\title{
Electrostatic Interaction across a Single-Layer Carbon Shell
} \author{
and T. Greber* ${ }^{*} \dagger$ \\ ${ }^{\dagger}$ Physik-Institut, Universität Zürich, CH-8057 Zürich, Switzerland \\ "Département de Chimie, École Normale Supérieure, F-75005 Paris, France \\ "Leibniz Institute of Solid State and Materials Research, D-01069 Dresden, Germany \\ ${ }^{\S}$ Paul Scherrer Institut, CH-5232 Villigen, Switzerland
}

R. Stania, ${ }^{\dagger}$ A. P. Seitsonen, ${ }^{\ddagger}$ D. Kunhardt, ${ }^{\text {IL }}$ B. Büchner, ${ }^{\text {IL }}$ A. A. Popov, ${ }^{\text {I॰ }}$ M. Muntwiler, ${ }^{\S}$

Supporting Information

ABSTRACT: Ions inside of fullerene molecules are model systems for the study of the electrostatic interaction across a single layer of carbon. For $\mathrm{TbSc}_{2} \mathrm{~N} @ \mathrm{C}_{80}$ on $h$-BN/ $\mathrm{Ni}(111)$, we observe with high-resolution X-ray photoelectron spectroscopy a splitting of the $\mathrm{C}$ 1s core level. The data may be explained quantitatively with density functional theory. The correlation of the $\mathrm{C} 1 \mathrm{~s}$ eigenvalues and the Coulomb potential of the inside ions at the corresponding carbon sites indicates incomplete screening of the electric field due to the endohedral ions. The screening comprises anisotropic charge transfer to the carbon atoms and their polarization. This behavior is essential for the ordering of endohedral single-molecule magnets and is expected to occur in any single-layer material.

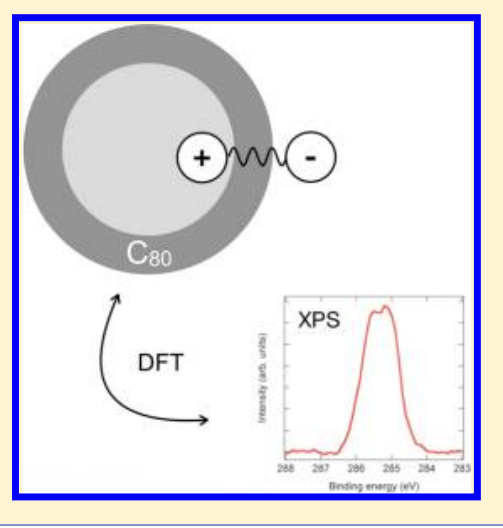

$\mathrm{E}$ ndohedral fullerene molecules are carbon cages such as $\mathrm{C}_{60}$ or $\mathrm{C}_{80}$ that contain atoms. In the case of encapsulated paramagnetic atoms, long spin coherence times ${ }^{1}$ and singlemolecule magnetism have been observed. ${ }^{2}$ The answer to the question of whether fullerenes match the picture of a Faraday cage $^{3}$ is important if, for example, the endohedral ordering is to be understood or if the magnetism shall be addressed with electric fields. Generally, electrostatic interaction with any single layer, such as, for example, two-dimensional graphene, affects the properties and performance of the material, and the interaction may also propagate across a single layer, which is an ultimate membrane that separates atoms in two volumes. For example, in the case of fluorinated graphene, ${ }^{4}$ one carbon atom in the two-atom unit cell undergoes bond formation to fluorine in volume $\mathrm{A}$ and the other one to fluorine in volume $\mathrm{B}$. This is due to a strong covalent $\mathrm{C}-\mathrm{F}$ bond and the minimization of steric repulsion. Interaction across a single layer is also expected in the case of noncovalently bound ionic species if their charge and magnetic moment are not completely screened. In the following, we will investigate screening of electric fields of ions inside of $\mathrm{C}_{80}$. The dielectric constant is a descriptor of the screening of an electric field in a continuum that results in polarization of its surface. In graphene, a dielectric constant has been deduced from the cyclotron mass. It depends on the charge carrier density, and values from 2.2 to 4.9 have been inferred. ${ }^{5}$ For bilayer graphene, theory predicted a dielectric constant $\epsilon_{\perp}$ of $\sim 3{ }^{6}$ Strong local electric fields due to a point charge of an ion near atomically thin layers and the discreteness of the charges may, however, impose charge transfer, i.e., charging of the layer at the nanometer scale, which is different from the concept of polarization of a dielectric.

Endohedral fullerenes are model systems for the study of electrostatic interaction across a single layer of carbon, where the fullerene cage takes the role of the carbon layer and the endohedral unit acts as a source of the electric field. The fact that different endohedral clusters, such as $\mathrm{Tb}_{2} \mathrm{ScN} @ \mathrm{C}_{80}$ and $\mathrm{TbSc}_{2} \mathrm{~N} @ \mathrm{C}_{80}$, may be separated with high-pressure liquid chromatography and the ordering of endohedral units in single crystals prove that there is interaction across the cage. ${ }^{7,8}$ Further evidence for interaction across the cage or recognition of the interior and its conformation come from the observation of the preferential orientation of endohedral units on copper ${ }^{9}$ and on rhodium, where concomitant magnetic ordering was observed. $^{10}$

For $\mathrm{C}_{80}$, it is known that nominally six electrons have to be transferred to the carbon atoms for a stable molecule with a large gap between the highest occupied (HOMO) and the lowest unoccupied molecular orbital (LUMO). ${ }^{11}$ In $\mathrm{R}_{3} \mathrm{~N} @ \mathrm{C}_{80}$, where $\mathrm{R}$ is a trivalent rare earth atom, this charge is obtained from the $\mathrm{R}_{3}^{3+} \mathrm{N}^{3-}$ endohedral cluster. Here we show that splitting of the $\mathrm{C} 1 \mathrm{~s}$ core level of $\mathrm{TbSc}_{2} \mathrm{~N} @ \mathrm{C}_{80}$ in highresolution X-ray photoelectron spectroscopy (XPS) is related to the presence of endohedral ions. The XPS data are quantitatively explained with density functional theory (DFT)

Received: April 26, 2018

Accepted: June 14, 2018

Published: June 14, 2018 
calculations that yield lower C 1s eigenvalues for carbon atoms close to positively charged endohedral ions. The eigenvalues are correlated with the Coulomb potential of the endohedral unit at the carbon sites, which imposes an anisotropic Coulomb potential outside of the carbon cages.

Figure 1a shows the Coulomb potential of a charge near a dielectric $(\epsilon=3) .{ }^{12}$ The dielectric becomes polarized, though

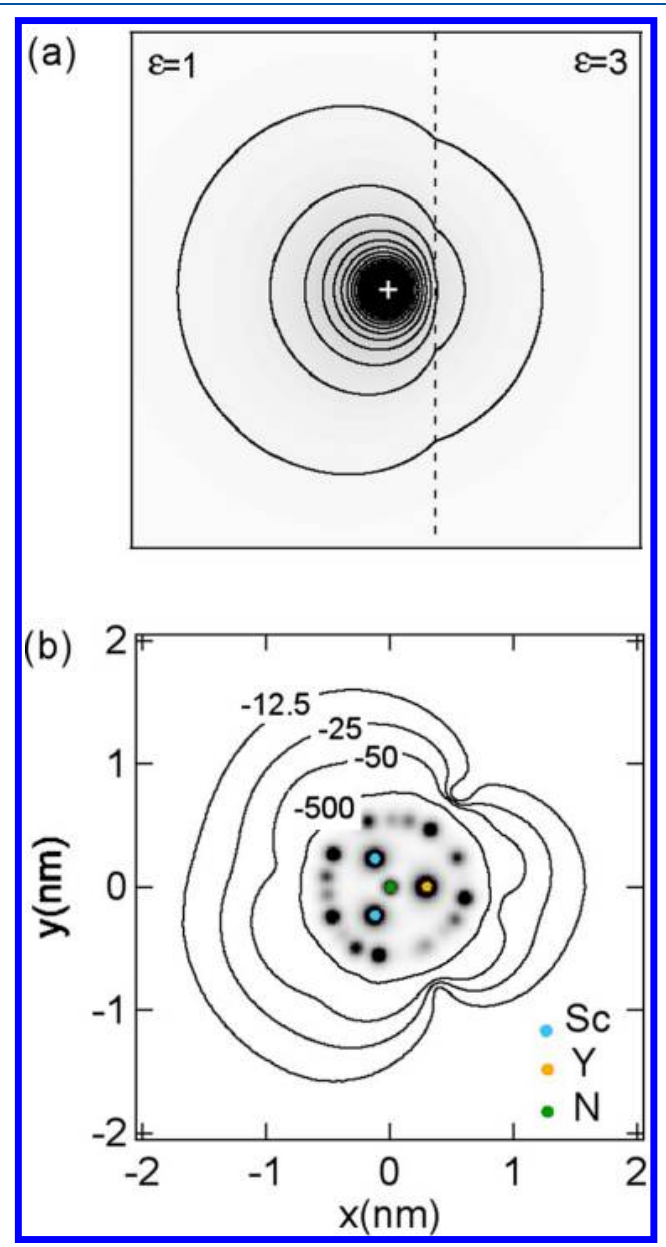

Figure 1. Electrostatic interaction across a dielectric interface. (a) Charge $(+)$ in the vacuum $(\epsilon=1)$ in front of a dielectric $(\epsilon=3)$. The contours represent the isopotentials in equal steps. The potential of the charge propagates into the dielectric and, if the latter is a layer, across it. (b) Coulomb potential of $\mathrm{YSc}_{2} \mathrm{~N} @ \mathrm{C}_{80}$ on the plane given by the $\mathrm{YSc}_{2}$ unit. The field exceeds the sphere that contains all molecular charge. The coordinate origin is set at the nitrogen nucleus (green). The rare earth ions are colored orange and light blue, and the carbon atoms lie on a perimeter with a $0.42 \mathrm{~nm}$ radius. The isopotential contours are referred to the potential at infinity and given in $\mathrm{meV}$.

some field of the ion propagates into the dielectric, as can be seen from the electrostatic isopotential contours. In a metal, where $\epsilon \rightarrow \infty$, the screening is complete and the situation of an ideal Faraday cage is met. At this stage, it is not clear to which extent this picture of a continuum model describes the situation of a charge close to a single-atom layer material. The discreteness of the charges and the atomic lattice may involve polarization in the form of an induced dipole and different charging of the carbon atoms that was predicted theoretically. $^{13}$

Figure $1 \mathrm{~b}$ displays the map of the electrostatic potential of $\mathrm{YSc}_{2} \mathrm{~N} @ \mathrm{C}_{80}$ in the endohedral plane as calculated with DFT.
We did the calculations with yttrium instead of terbium because $\mathrm{Y}$ has the same valence, very similar size, and electronegativity as $\mathrm{Tb}$, but $\mathrm{Y}$ results are, due to the absence of a partially filled $4 \mathrm{f}$ shell, more reliable (cf. the Methods section). Yttrium and scandium produce a different electrostatic potential outside of the molecule. This information helps to understand the crystallization behavior of the molecules ${ }^{8}$ and the interaction of the endohedral unit across the cage, which opens ways to electrostatically influence or control the endohedral orientation and magnetism. ${ }^{10}$

Figure 2 shows the comparison between the electrostatic potential at the positions of the 80 carbon atoms (a) and the

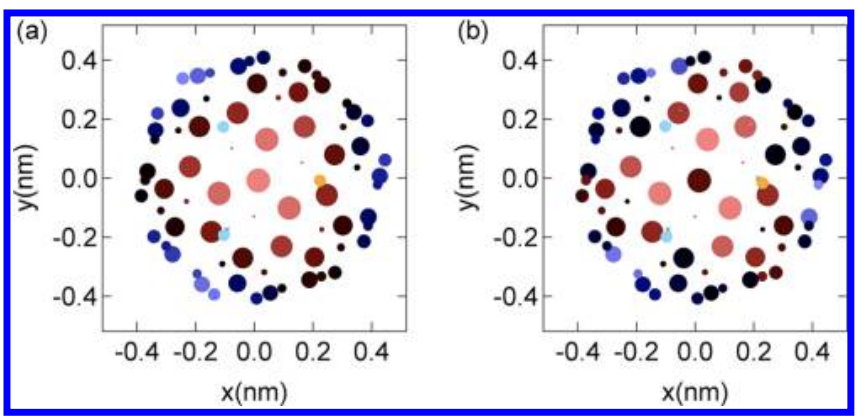

Figure 2. Comparison of the (a) Coulomb potential of the $\mathrm{YSc}_{2} \mathrm{~N}$ endohedral unit at the 80 carbon sites and (b) calculated $\mathrm{C} 1 \mathrm{~s}$ eigenvalues in $\mathrm{YSc}_{2} \mathrm{~N} @ \mathrm{C}_{80}$ (red, black, blue circles). Blue sites attract electrons most. The size of the circles represents the $z$-coordinate and renders a perspective. As in Figure 1, the endohedral atoms $\mathrm{Y}(\mathrm{Sc})$ are depicted as orange (light blue) dots.

corresponding C 1 s eigenvalues of $\mathrm{YSc}_{2} \mathrm{~N} @ \mathrm{C}_{80}$ (b). The atomic coordinates are taken from the DFT calculation. The Coulomb potential is calculated from three nominal $+3 e$ charges at the positions of the rare earth ions and one $-3 e$ charge at the site of the central nitrogen ion. From comparison of panels (a) and (b) in Figure 2, it becomes clear that the electrostatic potential shows the same trend as the eigenvalues: the carbon atoms in the vicinity of the rare earth ions have a lower electrostatic potential and lower eigenvalues. In order to compare the Coulomb potential of the endohedral unit $U$ at the atomic carbon sites and the $\mathrm{C} 1 \mathrm{~s}$ eigenvalues quantitatively, they are plotted in Figure $3 \mathrm{a}$, and the correlation is evident. The eigenvalues have a spread $\Delta E$ of $1.01 \mathrm{eV}$. They reflect the electrostatic potential after screening due to polarization of the carbon shell from different charges on the carbon atoms and possible variations due to the two different carbon species in $\mathrm{C}_{80}$. The majority species $\left(\mathrm{C}_{566}\right.$ or corannulene carbon $)$ is also encountered in $\mathrm{C}_{60}$ : 60 atoms sit on vertices where one pentagon and two hexagon faces meet. The minority species ( $\mathrm{C}_{666}$ or pyrene carbon) consists of 20 atoms that sit on vertices where three hexagons meet (see inset in Figure 3a). ${ }^{14}$ Both the $\mathrm{C}_{566}$ and the $\mathrm{C}_{666}$ eigenvalues follow the Coulomb potential, where we find a slope $\Delta E: \Delta U$ of 0.15 . The Coulomb potential variation $\Delta U$ of the nominal ionic charges in the cage is stronger than that of the eigenvalues $\Delta E$. As we see from the Bader charge analysis ${ }^{15}$ on all 84 atoms of the molecule, the DFT results produce, on average, about 0.6 times smaller charging than the nominal assignment (e.g., +3 for $\mathrm{Sc}$ or -3 for $\mathrm{N}) .{ }^{16}$ Furthermore, the screening of the potential at the carbon sites, i.e., the variation of the $C 1$ s eigenvalues, is affected by polarization and different charge transfer on the individual carbon atoms. In Figure 3b, the Bader charge on the 80 carbon 


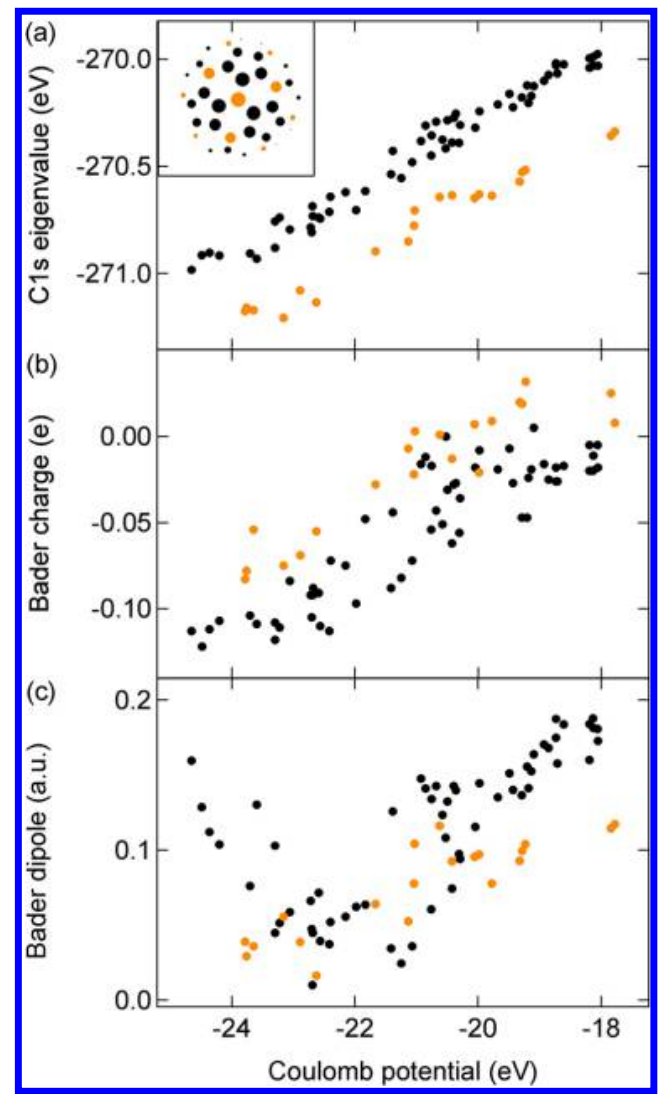

Figure 3. Correlation of the $\mathrm{C} 1 \mathrm{~s}$ eigenvalues (a), the Bader charges on the carbon atoms (b), and the Bader dipole moments in atomic units (c), with the nominal Coulomb potential $U$ of the endohedral unit on the positions of the 80 carbon atoms in $\mathrm{YSc}_{2} \mathrm{~N} @ \mathrm{C}_{80}$. The black dots represent the 60 corannulene carbons $\mathrm{C}_{566}$ and the orange dots the 20 pyrene carbons $\mathrm{C}_{666}$ (see inset in (a)). (a) Eigenvalue calculations predict an average chemical shift of $343 \mathrm{meV}$ between $\mathrm{C}_{566}$ and $\mathrm{C}_{666}$. (b) Bader charges indicate different electron affinities for corannulene and pyrene carbon. (c) The six corannulene carbons with low Coulomb potential have relatively large dipoles and signal the onset of chemical interaction.

atoms is plotted vs the Coulomb potential. The average Bader charge of $-0.045 e$ per carbon atom is scattered in an interval of $0.2 \mathrm{e}$. This scatter indicates the significant electron reallocation on the carbon cage, which is related to the Coulomb potential of the endohedral ions. The offset between the $\mathrm{C}_{566}$ and the $\mathrm{C}_{666}$ eigenvalues of $343 \mathrm{meV}$ is a chemical shift of different carbon species, where the lower eigenvalues of $\mathrm{C}_{666}$ are connected to a lower energy of the planar (pyrene) carbon. Although the lateral charge transfer on the carbon atoms does not support a classical polarization picture, we are able to estimate an effective dielectric constant of the cage material from

$$
\epsilon=\frac{n e}{2 \epsilon_{0}} \frac{\Delta p}{\Delta E}
$$

where $n$ is the areal dipole density on the carbon sphere, $e$ the elementary charge, $\epsilon_{0}$ the vacuum permittivity, and $\Delta p / \Delta E$ the slope of the dipole $p$ vs eigenvalue data. We recall that this assumes the eigenvalues to follow the true electrostatic potential at the carbon cores, and from eq 1 and all carbon atoms, we get an effective $\epsilon$ of $2.0 \pm 0.2$. The Bader dipole vs eigenvalue data show two more things: (i) The dipoles of the six carbon atoms $\left(C_{c}\right)$ closest to the endohedral ions do not follow the trend of the others because chemical interaction sets in and (ii) the corannulene carbons without the $C_{c}$ 's have a $70 \%$ larger effective $\epsilon$ than the pyrene carbons.

So far, we have discussed theoretical results of the electric screening of the ions. Because the $\mathrm{C} 1 \mathrm{~s}$ eigenvalues are reflected as well in core level photoemission binding energies, their comparison will test the theory. Figure 4 shows the C 1 s

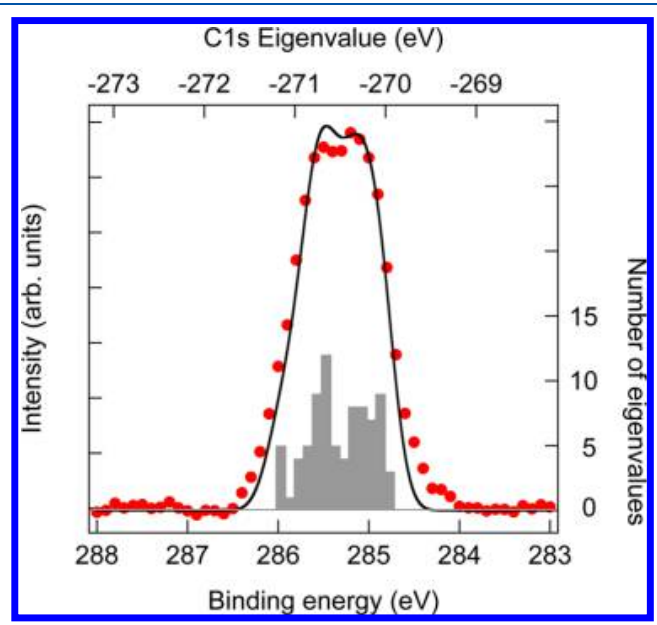

Figure 4. Comparison between calculated $\mathrm{C} 1 \mathrm{~s}$ eigenvalues of $\mathrm{YSc}_{2} \mathrm{~N} @ \mathrm{C}_{80}$ (gray bars and black line) and the high-resolution XPS data of one monolayer of $\mathrm{TbSc}_{2} \mathrm{~N} @ \mathrm{C}_{80}$ on $h-\mathrm{BN} / \mathrm{Ni}(111)$ (red dots). The eigenvalues span an energy range of $1.01 \mathrm{eV}$ and are grouped in bins of $100 \mathrm{meV}$. The black line was obtained by convoluting the eigenvalues with a Gaussian of $380 \mathrm{meV}$ fwhm. The eigenvalues are offset to the experimental binding energies (by $14.8 \mathrm{eV}$ ), which accounts for the $\mathrm{C}$ 1s hole in the photoemission final state.

eigenvalue spectrum of $\mathrm{YSc}_{2} \mathrm{~N} @ \mathrm{C}_{80}$ and high-resolution XPS data of the C 1s core level of a monolayer of $\mathrm{TbSc}_{2} \mathrm{~N} @ \mathrm{C}_{80}$ on a hexagonal boron nitride nickel $(h-\mathrm{BN} / \mathrm{Ni}(111))^{17}$ surface. In the Methods section, we give arguments why $\mathrm{Y}$ and $\mathrm{Tb}$ are considered to be equivalent for the present matters. Two peaks with an approximate 1:1 weight ratio are observed. This does not reflect the 3:1 ratio between $\mathrm{C}_{566}$ and $\mathrm{C}_{666}$ as seen in nuclear magnetic resonance ${ }^{14}$ but has its origin in the charge distribution of the endohedral cluster. The eigenvalues were convoluted with a Gaussian of $380 \mathrm{meV}$ full width at halfmaximum (fwhm) to account for the finite $\mathrm{C} 1 \mathrm{~s}$ lifetime and the experimental resolution. This is in line with $\mathrm{C}_{60}$ where at room temperature the $C$ 1s level has a fwhm of about 350 meV. ${ }^{18}$ Furthermore, the theoretical energy scale is offset by $14.8 \mathrm{eV}$ in order to match the experimental binding energies. This value accounts for the hole on the $C 1$ s shell, which is left behind in the photoemission process but is not considered in the present ground-state calculations. The agreement between theory and experiment is excellent, in particular, the experimentally observed C $1 \mathrm{~s}$ splitting is quantitatively reproduced. A posteriori this implies that $\mathrm{Y}$ and $\mathrm{Tb}$ must produce very similar electrostatics and that there are no strong additional energy differences among the 80 different photoemission final states. At this stage of comparison between experiment at $300 \mathrm{~K}$ and theory at $0 \mathrm{~K}$, the endohedral rotation ${ }^{19}$ that sets in at around $50 \mathrm{~K}^{20}$ does not impose a big change in the $\mathrm{C} 1 \mathrm{~s}$ eigenvalue distribution. In order to support this assessment, we show in the Supporting Information eigenvalue spectra of three different endohedral conformations that are expected to be populated at room temperature. 
The agreement between experiment and theory (Figure 4) and the correlation betweenthe Coulomb potential and $\mathrm{C} 1 \mathrm{~s}$ eigenvalues (Figure 3a) demonstrates that the $\mathrm{C}$ 1s core level energy contains information on the interaction potential of ions in and across single-layer carbon membranes.

In conclusion, we traced back the peak splitting in the $\mathrm{C} 1 \mathrm{~s}$ $\mathrm{X}$-ray photoemission spectrum of $\mathrm{TbSc}_{2} \mathrm{~N} @ \mathrm{C}_{80}$ on $h$-BN/ $\mathrm{Ni}(111)$ to the $\mathrm{C} 1 \mathrm{~s}$ eigenvalue distribution as obtained from DFT calculations. It was shown that the eigenvalues are correlated with the Coulomb potential of the endohedral unit. Analysis of the DFT results indicates laterally different charge distribution on the cage, but an average effective dielectric constant $\epsilon$ of about 2 may be inferred, which identifies $\mathrm{C}_{80}$ as a nonideal Faraday cage. From these findings, we predict significant nonisotropic electric fields outside of the molecule or behind any single-layer membrane with finite $\epsilon$ and with adsorbed ions on one side. In the present case, the electric fields are related to the endohedral cluster orientation, and they may be used for detecting and possibly addressing the endohedral orientation and magnetization.

\section{METHODS}

Theory. Because $\mathrm{Tb}^{3+}$ and $\mathrm{Y}^{3+}$ have the same formal charge and very similar Shannon ionic radii (0.923 and 0.9 $\AA$ ) and Pauling electronegativities (1.2 and 1.36), the DFT calculations were performed on the $\mathrm{YSc}_{2} \mathrm{~N} @ \mathrm{C}_{80}$ endofullerene ${ }^{21}$ rather than the lanthanide-containing $\mathrm{TbSc}_{2} \mathrm{~N} @ \mathrm{C}_{80}$ because magnetic interaction may be neglected for the atomic structure and yttrium is easier to handle theoretically and leads to more reliable results with the present-day approximations to the exchangecorrectional term in the Kohn-Sham single-particle equations. The calculations on the isolated $\mathrm{YSc}_{2} \mathrm{~N} @ \mathrm{C}_{80}$ cluster were performed with the TurboMole code, ${ }^{22}$ and details are given in the Supporting Information. ${ }^{16}$ They yield several possible orientations of the endohedral unit with very similar energies. ${ }^{23}$ We checked the $C_{3}, C_{s, a}$, and $C_{s, b}$ in vacuum structures of $\mathrm{YSc}_{2} \mathrm{~N} @ \mathrm{C}_{80 .}{ }^{16}$ In all three configurations, the endohedral $\mathrm{YSc}_{2} \mathrm{~N}$ unit is flat, i.e., the sum of the three pyramidal angles $\mathrm{Sc}_{\mathrm{A}}-\mathrm{N}-\mathrm{Sc}_{\mathrm{B}}, \mathrm{Sc}_{\mathrm{A}}-\mathrm{N}-\mathrm{Y}$, and $\mathrm{Y}-\mathrm{N}-\mathrm{Sc}_{\mathrm{B}}$ is $360^{\circ} .{ }^{16}$ In the main text, we show the results for the lowest-energy $C_{3}$ structure.

Experimental Section. $\mathrm{TbSc}_{2} \mathrm{~N} @ \mathrm{C}_{80}$ with icosahedral $I_{h}$ symmetry was synthesized and purified as described in refs 8 and 24 and sublimated onto $h-\mathrm{BN} / \mathrm{Ni}(111) .{ }^{17}$ As for $\mathrm{C}_{60}{ }^{25}$ this substrate does not impose strong binding of the molecules to the substrate. The normal emission XPS data were recorded at room temperature and a photon energy of $600 \mathrm{eV}$. The overall energy resolution was below $200 \mathrm{meV}^{26}$ The coverage was determined with a layer by layer growth model with an electron mean free path of $1 \mathrm{~nm}$ and from the intensity ratio of the $\mathrm{N} 1 \mathrm{~s}$ core levels of the molecule and the hexagonal boron nitride substrate. ${ }^{16}$

\section{ASSOCIATED CONTENT}

\section{(5) Supporting Information}

The Supporting Information is available free of charge on the ACS Publications website at DOI: 10.1021/acs.jpclett.8b01326.

Details of density functional theory calculations, coordinates and eigenvalues of $\mathrm{YSc}_{2} \mathrm{~N} @ \mathrm{C}_{80}$, structures $C_{3}, C_{s, a}$, and $C_{s, b}$, comparison of eigenvalue spectra $C_{3}$, $C_{s, a}, C_{s, b}$, details of the sample preparation, details of the measurements, and coverage determination with XPS (PDF)

\section{AUTHOR INFORMATION}

\section{Corresponding Author}

*E-mail: greber@physik.uzh.ch. ORCID

A. P. Seitsonen: 0000-0003-4331-0650

A. A. Popov: 0000-0002-7596-0378

T. Greber: 0000-0002-5234-1937

\section{Notes}

The authors declare no competing financial interest.

\section{ACKNOWLEDGMENTS}

This work was supported by the Swiss National Science Foundation Grant No. 200020 153312. Calculations were performed at the CSCS Swiss National Supercomputing Centre under Project uzh11. The photoemission measurements were performed at the PEARL beamline of the Swiss Light Source, Paul Scherrer Institut, Villigen. A.A.P. acknowledges the European Research Council (ERC) under the European Union Horizon 2020 research and innovation programme (Grant No. 648295 GraM3).

\section{REFERENCES}

(1) Almeida Murphy, T.; Pawlik, Th.; Weidinger, A.; Höhne, M.; Alcala, R.; Spaeth, J.-M. Observation of Atomlike Nitrogen in Nitrogen-Implanted Solid $\mathrm{C}_{60}$. Phvs. Rev. Lett. 1996, 77, 1075.

(2) Westerström, R.; Dreiser, J.; Piamonteze, C.; Muntwiler, M.; Weyeneth, S.; Brune, H.; Rusponi, S.; Nolting, F.; Popov, A.; Yang, S.; et al. An Endohedral Single-Molecule Magnet with Long Relaxation Times: DySc $\mathrm{N}_{2} @ \mathrm{C}_{80}$. I. Am. Chem. Soc. 2012, 134, 9840-9843.

(3) Delaney, P.; Greer, J. $\mathrm{C}_{60}$ as a Faraday cage. Appl. Phys. Lett. 2004, 84, 431-433.

(4) Sahin, H.; Topsakal, M.; Ciraci, S. Structures of fluorinated graphene and their signatures. Phys. Rev. B: Condens. Matter Mater. Phvs. 2011, 83, 115432.

(5) Elias, D. C.; Gorbachev, R. V.; Mayorov, A. S.; Morozov, S. V.; Zhukov, A. A.; Blake, P.; Ponomarenko, L. A.; Grigorieva, I. V.; Novoselov, K. S.; Guinea, F.; et al. Dirac cones reshaped by interaction effects in suspended graphene. Nat. Phvs. 2011, 7, 701704.

(6) Santos, E. J. G.; Kaxiras, E. Electric-Field Dependence of the Effective Dielectric Constant in Graphene. Nano Lett. 2013, 13, 898902.

(7) Popov, A. A.; Yang, S.; Dunsch, L. Endohedral Fullerenes. Chem. Rev. 2013, 113, 5989-6113.

(8) Stevenson, S.; Chancellor, C. J.; Lee, H. M.; Olmstead, M. M.; Balch, A. L. Internal and External Factors in the Structural Organization in Cocrystals of the Mixed-Metal Endohedrals (GdSc $c_{2} \mathrm{~N} @ \mathrm{I}_{h}-\mathrm{C}_{80}, \mathrm{Gd}_{2} \mathrm{ScN} @ \mathrm{I}_{h}-\mathrm{C}_{80}$ and $\mathrm{TbSc}_{2} \mathrm{~N} @ \mathrm{I}_{h}-\mathrm{C}_{80}$ ) and Nickel(II) Octaethylporphyrin. Inorg. Chem. 2008, 47, 1420-1427.

(9) Treier, M.; Ruffieux, P.; Fasel, R.; Nolting, F.; Yang, S.; Dunsch, L.; Greber, T. Looking inside an endohedral fullerene: Inter- and intramolecular ordering of $\mathrm{Dy}_{3} \mathrm{~N} @ \mathrm{C}_{80}\left(\mathrm{I}_{h}\right)$ on $\mathrm{Cu}(111)$. Phvs. Rev. B: Condens. Matter Mater. Phvs. 2009, 80, 081403.

(10) Westerström, R.; Uldry, A. C.; Stania, R.; Dreiser, J.; Piamonteze, C.; Muntwiler, M.; Matsui, F.; Rusponi, S.; Brune, H.; Yang, S.; et al. Surface Aligned Magnetic Moments and Hysteresis of an Endohedral Single-Molecule Magnet on a Metal. Phys. Rev. Lett. 2015, 114, 087201 .

(11) Nakao, K.; Kurita, N.; Fujita, M. Ab initio molecular-orbital calculation for $\mathrm{C}_{70}$ and seven isomers of $\mathrm{C}_{80}$. Phvs. Rev. B: Condens. Matter Mater. Phvs. 1994, 49, 11415-11420. 
(12) Jackson, J. D. Classical electrodynamics, 3rd ed.; Wiley: New York, 1999.

(13) Popov, A. A.; Kaestner, C.; Krause, M.; Dunsch, L. Carbon Cage Vibrations of $\mathrm{M} @ \mathrm{C}_{82}$ and $\mathrm{M}_{2} @ \mathrm{C}_{2 n}(\mathrm{M}=\mathrm{La}, \mathrm{Ce} ; 2 n=72,78$, 80): The Role of the Metal Atoms. Fullerenes, Nanotubes, Carbon Nanostruct. 2014, 22, 202-214.

(14) Stevenson, S.; Rice, G.; Glass, T.; Harich, K.; Cromer, F.; Jordan, M. R.; Craft, J.; Hadju, E.; Bible, R.; Olmstead, M. M.; et al. Small-bandgap endohedral metallofullerenes in high yield and purity. Nature 1999, 401, 55-57.

(15) Bader, R. F. W. Atoms in Molecules - A Quantum Theory; International series of monographs on chemistry; Oxford University Press: Oxford, U.K., 1990.

(16) See the Supporting Information: DFT total energy, coordinates, $\mathrm{C} 1 \mathrm{~s}$ eigenvalues, Bader charges and dipoles for $C_{3}$, the total energies and coordinates of the $C_{s, a}$ and $C_{s, b}$ low-energy structures, and experimental details.

(17) Auwärter, W.; Kreutz, T. J.; Greber, T.; Osterwalder, J. XPD and STM investigation of hexagonal boron nitride on $\mathrm{Ni}(111)$. Surf. Sci. 1999, 429, 229-236.

(18) Goldoni, A.; Cepek, C.; Larciprete, R.; Sangaletti, L.; Pagliara, S.; Paolucci, G.; Sancrotti, M. Core Level Photoemission Evidence of Frustrated Surface Molecules: A Germ of Disorder at the (111) Surface of $C_{60}$ before the Order-Disorder Surface Phase Transition. Phvs. Rev. Lett. 2002, 88, 196102.

(19) Fu, W.; Wang, X.; Azuremendi, H.; Zhang, J.; Dorn, H. C. 14N and $45 \mathrm{Sc}$ NMR study of trimetallic nitride cluster (M3N)6+ dynamics inside a icosahedral C80 cage. Chem. Commun. 2011, 47, 3858.

(20) Kostanyan, A.; Westerström, R.; Zhang, Y.; Kunhardt, D.; Stania, R.; Büchner, B.; Popov, A. A.; Greber, T. Switching Molecular Conformation with the Torque on a Single Magnetic Moment. Phvs. Rev. Lett. 2017, 119, 237202.

(21) Chen, N.; Fan, L.-Z.; Tan, K.; Wu, Y.-Q.; Shu, C.-Y.; Lu, X.; Wang, C.-R. Comparative spectroscopic and reactivity studies of $\mathrm{Sc}_{3-x} \mathrm{Y}_{x} \mathrm{~N} @ \mathrm{C}_{80}(x=0-3)$. L.Phvs. Chem. C 2007, 111, 11823-11828.

(22) Ahlrichs, R.; Bar, M.; Haser, M.; Horn, H.; Kolmel, C. Electronic-Structure Calculations on Workstation Computers - The Program System TURBOMOLE. Chem. Phvs. Lett. 1989, 162, 165169.

(23) Yang, S.; Popov, A. A.; Dunsch, L. Carbon Pyramidalization in Fullerene Cages Induced by the Endohedral Cluster: Non-Scandium Mixed Metal Nitride Clusterfullerenes. Angew. Chem. Int. Ed. 2008, 47, 8196-8200.

(24) Zhang, Y.; Krylov, D.; Rosenkranz, M.; Schiemenz, S.; Popov, A. A. Magnetic anisotropy of endohedral lanthanide ions: paramagnetic NMR study of $\mathrm{MSc}_{2} \mathrm{~N} @ \mathrm{C}_{80}$-Ih with $\mathrm{M}$ running through the whole 4 f row. Chem. Sci. 2015, 6, 2328-2341.

(25) Muntwiler, M.; Auwärter, W.; Seitsonen, A. P.; Osterwalder, J.; Greber, T. Rocking-motion-induced charging of $\mathrm{C}_{60}$ on $h-\mathrm{BN} /$ $\mathrm{Ni}(111)$. Phys. Rev. B: Condens. Matter Mater. Phvs. 2005, 71, 121402. (26) Muntwiler, M.; Zhang, J.; Stania, R.; Matsui, F.; Oberta, P.; Flechsig, U.; Patthey, L.; Quitmann, C.; Glatzel, T.; Widmer, R.; et al. Surface science at the PEARL beamline of the Swiss Light Source. I. Sunchrotron Radiat. 2017, 24, 354. 